\title{
DIVISIONS IV-V / WORKING GROUP Ap \& RELATED STARS
}

\author{
CHAIR \\ PAST CHAIR \\ BOARD
}

\author{
Gautier Mathys \\ Margarida Cunha \\ Michael Dworetsky \\ Oleg Kochukhov \\ Friedrich Kupka \\ Francis LeBlanc \\ Richard Monier \\ Ernst Paunzen \\ Olga Pintado \\ Nikolai Piskunov \\ Jozef Ziznovsky
}

\section{TRIENNIAL REPORT 2009-2012}

\section{Scientific highlights}

The purpose of the Working Group on Ap and Related Stars (ApWG) is to promote and facilitate research about stars in the spectral type range from $B$ to early $F$ that exhibit surface chemical peculiarities and related phenomena. This is a very active field of research, in which a wide variety of new developments have taken place since 2009, as illustrated by the following selected highlights.

The evolutionary context of the large-scale organised magnetic fields of Ap stars, which have been known for more than 60 years to be one of their most salient features, is starting to be outlined with the recent detection and study of rotationally modulated magnetic fields in their progenitors, the Herbig Ae/Be stars (Alecian et al. 2009; Hubrig et al. 2011a) and the identification and characterisation of magnetic late-type supergiants that are their potential descendants (Grunhut et al. 2010; Aurière et al. 2011). On the other hand, the sequence of hotter early-type magnetic stars is becoming increasingly populated through the works of the MiMeS (Magnetism in Massive Stars) collaboration (e.g., Wade et al. 2011) and of other teams (e.g., Hubrig et al. 2011b), putting the magnetism of Ap stars in a new perspective. The discovery of sub-Gauss magnetic fields with large-scale structure in the A0V star Vega (Lignières et al. 2009; Alina et al. 2011) and in the hot Am star Sirius (Petit et al. 2011) raises the possibility that all tepid main-sequence stars may be magnetic to a certain level. However, unprecedented stringent limits have been set on the mean longitudinal magnetic fields of Am and HgMn stars (e.g., Aurière et al. 2010; Makaganiuk et al. 2011), indicating that they must be at least one order of magnitude weaker than those of magnetic Ap stars.

Arlt \& Rüdiger (2010) have proposed Tayler instability as a possible mechanism of generation of magnetic fields in A stars while Ferrario et al. (2009) have suggested that magnetic Ap stars could have formed from the merging of two protostars.

Kepler observations have allowed the non-radial pulsation modes of hundreds of A-F stars to be classified, revealing the existence of a class of "hybrid" stars showing both 
$\gamma$ Dor and $\delta$ Sct pulsations (Uytterhoeven et al. 2011). They have also led to the first detections of $\gamma$ Dor and $\delta$ Sct pulsations in Ap stars; in particular, the A5p star KIC 8677585 is the first star to show both the rapid oscillations typical of Ap stars and $\gamma$ Dor pulsations (Balona et al. 2011). $\delta$ Sct and $\gamma$ Dor pulsations have been found in about $200 \mathrm{Am}$ stars from the analysis of SuperWASP observations (Smalley et al. 2011); such pulsations were also studied with Kepler (Balona et al. 2011). A new rapidly oscillating Ap (roAp) star discovered with Kepler, KIC 10195926, has been found to pulsate in two modes with different axes, unlike any roAp star known so far (Kurtz et al. 2011). These results can be expected to represent only the first steps of a major breakthrough in our knowledge of the pulsational properties of tepid stars, made possible in particular by the unprecedented amount of data that are becoming available from the space missions CoRoT and Kepler. In parallel, the pioneering satellite MOST continues to deliver datasets allowing the pulsations of rapidly oscillating Ap (roAp) stars to be studied in great detail (e.g., Gruberbauer et al. 2011). Bigot \& Kurtz (2011) have computed theoretical light curves of such stars in the framework of the revisited oblique pulsator model, matching well the observations. Khomenko \& Kochukhov (2009) have performed simulations of magnetoacoustic pulsations in their atmospheres. Sousa \& Cunha (2011) have shown that $\pi \mathrm{rad}$ jumps that have been seen in the pulsation phase of some spectral lines of roAp stars, may be due to the fact that that their surface is not spatially resolved in the observations rather than reflect the presence of a pulsation node in their outermost layers.

Additional observational results worth noting include the determination of the angular diameter of the Ap star $\gamma \mathrm{Equ}$ (one of the prototypes of the class) and derivation of its fundamental parameters from interferometric observations (Perraut et al. 2011), the observation of debris disks around main-sequence A stars (Smith \& Wyatt 2011), the first spectroscopic verification of an extragalactic Ap star (Paunzen et al. 2011), the investigation of the correlation of the surface brightness distribution of an Ap star (as inferred from CoRoT observations) with its surface chemical abundance inhomogeneities and its magnetic field (Lüftinger et al. 2010), the inference from the analysis of spectropolarimetric observations of surprisingly complex magnetic structures in the Ap star $\alpha^{2}$ CVn (Kochukhov \& Wade 2010) and in the Bp star HD 37776 (Kochukhov et al. 2011), and the characterisation of the long-term modulation of the rotation periods of Ap and Bp stars (Mikulášek et al. 2011).

On the theoretical front, Vick et al. (2010) have developed self-consistent evolution models of Am stars including atomic diffusion and mass loss. Théado et al. (2009) have demonstrated the importance of thermohaline convection in the computation of the abundance variations induced by atomic diffusion. Model atmospheres including abundance stratification at equilibrium have been constructed by Alecian \& Stift (2010) and LeBlanc et al. (2009). Alecian et al. (2011) have computed numerical simulations of time-dependent diffusion in stellar atmospheres. Stift et al. (2011) have illustrated the importance of taking into account variations of the structure of the atmospheres across the stellar surface for mapping of abundance inhomogeneities on the surface of Ap stars.

\section{The role of the Working Group on Ap and Related Stars}

The above summary is by no means exhaustive. But it shows how the theme of the Working Group transcends the borders between various fields of astrophysics. It ranges across the subjects of a large number of IAU Commissions: in particular, stellar photometry and polarimetry (C25), variable stars (C27), stellar spectra (C29), stellar constitution (C35), and theory of stellar atmospheres (C36). Ap and related stars display the most extreme manifestations of stellar physical processes such as magnetism and 
selective diffusion, and their understanding requires the consideration of a broad spectrum of mechanisms, including stellar pulsation, radiative acceleration and winds, accretion, and binarity. The role of the ApWG in providing a meeting point and a channel of communication for the members of a community involving such a wide range of scientific expertise, is essential. One of the ways in which the WG fulfils this role is via its ApN newsletter (http://apn.arm.ac.uk/newsletter/). This newsletter is used both to announce news and as a public forum, where registered members can submit requests or announcements, and initiate discussions or participate in them.

The ApWG also serves as a contact point with the atomic physics community, to compile and communicate to the latter requests for new atomic data that are needed for the analysis and modelling of Ap and related stars.

\section{Scientific meetings}

To follow up on the two science meetings that it organised during the previous triennium ( CP\#Ap Workshop in Vienna, Austria, in 2007 and Joint Discussion on Progress in understanding the physics of $A p$ and related stars at the IAU XXVIIth General Assembly in Rio de Janeiro, Brazil, in 2009), the ApWG has now started plans for a symposium entitled Putting A Stars into Context, to take place in 2013. A number of other conferences and workshops including sessions relevant to the physics and study of Ap and related stars took place or will take place between August 2009 and August 2012. They include: Seismological Challenges for Stellar Structure (Lanzarote, Spain, February 2010); Magnetic Fields From Core Collapse to Young Stellar Objects (London, Ontario, Canada, May 2010); IAU Symposium 272: Active OB Stars: Structure, Evolution, Mass Loss and Critical Limits (Paris, France, July 2010); 10th International Colloquium on Atomic Spectra and Oscillator Strengths for Astrophysical and Laboratory Plasmas (Berkeley, California, USA, August 2010); IAU Symposium 273: Physics of Sun and Star Spots (Ventura, California, USA, August 2010); Magnetic Stars 2010 (Nizhny Arkhyz, Russia, August 2010); Progress in Solar/Stellar Physics with Helio- and Asteroseismology (Hakone, Japan, March 2011); Stellar Polarimetry: From Birth to Death (Madison, Wisconsin, USA, June 2011); 4th Kepler Asteroseismic Science Consortium workshop: From unprecedented data to revolutionary science (Boulder, Colorado, USA, July 2011); 7th Potsdam Thinkshop: Magnetic Fields in Stars and Exoplanets (Potsdam, Germany, August 2011); The First Kepler Science Conference (Moffett Field, California, USA, December 2011). Members of the ApWG feature both as organisers and as invited speakers of these meetings.

\section{Conclusion}

The community interested in Ap and related stars has been very active scientifically over this triennium. Considerable progress has been made, opening new perspectives in several areas, such as magnetic fields (with the novel possibility to follow their evolution from pre- to post-main-sequence stages, the developing knowledge of their extension to hotter stars, and the realisation that they may be ubiquitously present in all A stars, with different strengths) and pulsation (with an upheaval of the overall picture of pulsation in A and B stars resulting from the unprecedented wealth of exquisitely detailed observations obtained e.g. by the space missions CoRoT and Kepler). Developments in these, and related fields, will assuredly continue in the next triennium, and the Working Group 
on Ap \& Related Stars will play a critical role in ensuring that effective interactions take place between the various constituents of this research community.

\author{
Gautier Mathys \\ chair of Working Group
}

\title{
References
}

Alecian, E., Wade, G. A., Catala, C., et al. 2009, MNRAS, 400, 354

Alecian, G. \& Stift, M. J. 2010, A\&A A, 516, A53

Alecian, G., Stift, M. J., \& Dorfi, E. A. 2011, MNRAS (in press)

Alina, D., Petit, P., Lignières, F., et al. 2011, arXiv:1107.5639

Arlt, R. \& Rüdiger, G. 2011, AN, 332, 70

Aurière, M., Wade, G. A., Lignières, F., et al. 2010, A\&\&A, 523, A40

Aurière, M., Konstantinova-Antova, R., Petit, P., et al. 2011, arXiv:1109.5570

Balona, L. A., Cunha, M. S., Kurtz, D. W., et al. 2011, MNRAS, 410, 517

Balona, L. A., Ripepi, V., Catanzaro, G., et al. 2011, MNRAS, 414, 792

Bigot, L. \& Kurtz, D. W. 2011, arXiv:1110.0988

Ferrario, L., Pringle, J. E., Tout, C. A., \& Wickramasinghe, D. T. 2009, MNRAS, 400, L71

Gruberbauer, M., Huber, D., Kuschnig, R., et al. 2011, A\&SA, 530, A135

Grunhut, J. H., Wade, G. A., Hanes, D. A., \& Alecian, E. 2010, MNRAS, 408, 2290

Hubrig, S., Mikulášek, Z., González, J. F., et al. 2011, A\&A, 525, L4

Hubrig, S., Schöller, M., Kharchenko, N. V., et al. 2011, A\&A, 528, A151

Khomenko, E. \& Kochukhov, O. 2009, ApJ, 704, 1218

Kochukhov, O. \& Wade, G. A. 2010, A\&A, 513, A13

Kochukhov, O., Lundin, A., Romanyuk, I., \& Kudryavtsev, D. 2011, ApJ, 726, 24

Kurtz, D. W., Cunha, M. S., Saio, H., et al. 2011, MNRAS, 414, 2550

LeBlanc, F., Monin, D., Hui-Bon-Hoa, A., \& Hauschildt, P. H. 2009, A\& A, 495, 937

Lignières, F., Petit, P., Böhm, T., \& Aurière, M. 2009, AछA, 500, L41

Lüftinger, T., Fröhlich, H.-E., Weiss, W. W., et al. 2010, A\&AA, 509, A43

Makaganiuk, V., Kochukhov, O., Piskunov, N., et al. 2011, A\&AA, 525, A97

Mikulášek, Z., Krtička, J., Henry, G. W., et al. 2011, A\&̇A, 534, L5

Paunzen, E., Netopil, M., \& Bord, D. J. 2011, MNRAS, 411, 260

Perraut, K., Brandão, I., Mourard, D., et al. 2011, A\&A, 526, A89

Petit, P., Lignières, F., Aurière, M., et al. 2011, A\&A, 532, L13

Smalley, B., Kurtz, D. W., Smith, A. M. S., et al. 2011, arXiv:1107.0246

Smith, R. \& Wyatt, M. C. 2011, A\&A, 515, A95

Sousa, J. C. \& Cunha, M. S. 2011, MNRAS, 414, 2576

Stift, M. J., Leone, F., \& Cowley, C. R. 2011, arXiv:1110.1291

Théado, S., Vauclair, S., Alecian, G., \& LeBlanc, F. 2009, ApJ, 704, 1262

Uytterhoeven, K., Moya, A., Grigahcene, A., et al. 2011, arXiv:1107.0335

Vick, M., Michaud, G., Richer, J., \& Richard, O. 2010, AछA, 521, A62

Wade, G. A., Howarth, I. D., Townsend, R. H. D., et al. 2011, MNRAS, 416, 3160 